\title{
Impact Du Conseil Agricole Sur Les Performances Des Producteurs D'anacarde De Cote d'Ivoire
}

\author{
Doukouré Charles Fe, PhD
}

Ecole Nationale Supérieure de Statistique et d'Economie Appliquée (ENSEA), Côte d'Ivoire, Cellule d'Analyse de Politiques Economiques du

Cires (CAPEC, Côte d'Ivoire

Kodjo Apollinaire Aklobessi, Ingénieur des Travaux

\section{Statistiques}

Ecole Nationale Supérieure de Statistique et d'Economie Appliquée

(ENSEA), Côte d'Ivoire

Doi: 10.19044/esj.2018.v14n30p292 URL:http://dx.doi.org/10.19044/esj.2018.v14n30p292

\begin{abstract}
The cashew sector has been booming in Côte d'Ivoire for the last three years. In the face of international competition, it appeared important to improve the performance of producers in order to maintain the comparative advantages of Côte d'Ivoire. In this perspective, the agricultural advisory project was set up to train cashew farmers in good practices. This study aims to evaluate the impact of this project on their performance in terms of returns. The data used for the analysis come from the survey conducted by the National Agency for Rural Development (ANADER). The average effect was estimated trough the propensity score matching method by using the kernel method. The results reveal that the advice and training support of cashew producers has led to a significant increase in yield of $22.4 \%$ on average.
\end{abstract}

Keywords: Impact evaluation, propensity score matching, kernel method, farmer yield

\section{Résumé}

La filière anacarde est en plein essor en Côte d'Ivoire depuis ces trois dernières années. Face à la concurrence internationale, il est apparu important d'améliorer les performances des producteurs dans le but de maintenir les avantages comparatifs de la Côte d'Ivoire. C'est dans cette perspective que le projet de conseil-agricole a été mis en place en vue de former les producteurs d'anacarde aux bonnes pratiques. Cette étude vise à évaluer l'impact de ce projet sur leur performance en terme de rendements. Les données utilisées pour l'analyse proviennent de l'enquête réalisée par l'Agence National pour 
le Développement Rural (ANADER). L'effet moyen a été estimé par la méthode d'appariement sur scores de propension. C'est la méthode du noyau qui a été retenue. Les résultats révèlent que l'appui conseil-formation des producteurs d'anacarde a induit une augmentation significative du rendement de $22,4 \%$ en moyenne.

Mots clés: Evaluation d'impact, appariement sur score de propension, méthode du noyau, rendements des producteurs

\section{Introduction}

Depuis ces cinq dernières années, l'anacarde occupe une place importante dans les performances du secteur agricole en Côte d'Ivoire. Cette culture est au centre des de plusieurs réformes pour permettre à la filière d'être plus dynamique dans le processus de développement de la Côte d'ivoire. La production de noix de cajou a cru de plus de $11 \%$ par an entre 2004 et 2014, faisant de la Côte d'Ivoire le premier producteur et exportateur mondial de noix brute devant l'Inde avec une production 2015 de 702510 tonnes. En 2017, cette production atteint 711236 tonnes (Conseil Coton Anacarde, 2018). Les superficies cultivées sont passées de 8.220 hectares en 1970 à environ 450.000 hectares en 2008 (Ministère de l'Intégration Africaine et des Ivoiriens de l'Extérieur, 2018). En outre, l'anacarde constitue en volume, le troisième produit d'exportation de la Côte d'Ivoire après le cacao et l'hévéa. Les réformes mises en place en 2002 et 2013 ont soutenu cette dynamique.

Cependant, la filière, est confrontée à de nombreux défis internes tels que le faible niveau de transformation locale et de diversification, le faible niveau de mécanisation qui limitent la productivité des parcelles. En outre . En plus de ces défis, l'on pourrait évoquer que plusieurs facteurs externes qui limitent la production et la productivité. Ce sont entre autres, le vieillissement du verger, la baisse de la fertilité des sols, les conditions de vie précaires des communautés productrices de noix d'anacarde, le retard de la production dû au phénomène climatique. Plusieurs investigations sont donc menées face à la pluralité des menaces pour améliorer la productivité du secteur agricole en générale et de la filière anacarde en particulier. Au nombre de ces réformes, la filière anacarde a connu une restructuration au niveau organisationnel, juridique et institutionnel avec la création en 2013 du Conseil de Régulation, de Suivi et de Développement des filières Coton et Anacarde (Conseil du Coton et de l'Anacarde), en remplacement de l'ARECA ${ }^{2}$. Au nombre des

${ }^{2}$ Le Conseil de régulation, de suivi et de développement des filières coton et anacarde en abrégé, le Conseil du Coton et de 1 'Anacarde est un organe créé par la loi n 2013-656 du septembre 2013 fixant les règles relatives à la commercialisation du coton et de l'anacarde et à la régulation des activités dans les filières coton et anacarde. 
actions réalisées dans le cadre de cette réforme, c'est l'accentuation de la formation des producteurs.

L'amélioration du niveau de connaissance des producteurs est somme toute une stratégie pour les autorités de la filière pour améliorer la performance des producteurs. Ainsi, le nombre de producteurs touchés par les formations est passé de 102282 en 2015 à 149950 en 2016 (Conseil Coton Anacarde, 2018). En effet l'un des points clés des réformes réside dans l'optimisation de la production et l'amélioration de la qualité. C'est dans cette optique qu'un accord cadre tripartite a été signé en 2014 entre le Conseil Coton-Anacarde, le Fonds Interprofessionnel pour la Recherche et le Conseil Agricole (FIRCA) et l'Agence Nationale d'Appui pour le Développement Rural (ANADER) pour assurer le conseil agricole et l'encadrement des producteurs d'anacarde en Côte d'Ivoire. Conformément à la convention signée, il était question de renforcer les capacités de l'ensemble des producteurs sur les bonnes pratiques agricoles en vue de leur permettre d'accroître durablement leur production d'anacarde. Le but de cette étude est d'évaluer l'impact de ce projet de conseil dédié aux producteurs d'anacarde en Côte d'Ivoire sur le rendement des parcelles.

La présente étude aiderait les pouvoirs publics, les principaux acteurs et les bailleurs de fonds du programme à évaluer les effets de cet appui conseil sur les performances des producteurs d'anacarde de Côte d'Ivoire. La question que l'on se pose est de savoir: est-ce que l'appui conseil a permis une amélioration des rendements des parcelles d'anacarde?

La suite de l'étude est organisée en quatre sections. La première section fait une revue de littérature sur les résultats de 1'appui conseil en agriculture sur les performances des producteurs. Les deuxième et troisième sections présentent respectivement une description du projet d'appui-conseil et l'approche méthodologique utilisée dans cette étude. La quatrième section s'intéresse aux résultats de l'évaluation avant la conclusion et les recommandations.

\section{Revue de littérature}

De nos jours avec la combinaison des facteurs tels que les difficultés d'accès à la technologie et à l'innovation, les contraintes de financement, la baisse de la fertilité des sols, les effets du changement climatique, le secteur agricole fait face à plusieurs défis notamment celui du maintien du niveau de la production dans les pays en développement. Selon une étude au Niger, dans le domaine agricole, la faiblesse des rendements des producteurs peut s'expliquer par le faible niveau d'encadrement de ces derniers et l'inaccessibilité aux innovations agricoles (MAG-SDR, 2010). Deux sources d'amélioration de la performance des producteurs apparaît ici : l'encadrement des producteurs donc la formation et l'accès à l'innovation. 
S'agissant de la formation, plusieurs auteurs montrent que cela consitute un recours presqu'idéal pour l'amléioration de la productivité des agriculteurs. Dans cette veine, pour Maïga et al, (2017) les sous performances des producteurs de riz au Niger sont dues à l'inefficience de l'encadrement technique. Cette inefficience qui s'explique par le faible taux de couverture des prestations d'encadrement, le manque de matériel technique et de moyens financiers. En outre cet encadrement est basé sur des méthodes traditionnelles pour la diffusion du conseil agricole. De prime abord, cet encadrement a un faible de taux de couverture et ensuite le personnel manque de moyens technique et financier qui n'arrive pas à toucher le plus grand nombre de producteurs. Selon eux, les méthodes appliquées actuellement dans la riziculture au Niger sont des méthodes dépassées et qu'il serait donc nécessaire que les méthodes de diffusion du conseil agricole soient révisées.

Aly et al, (2017) ont montré la formation des agriculteurs à l'usage des nouvelles variétés de semences a un impact positif sur le rendement des producteurs de Niébé au Bénin. En effet, selon les travaux de ces auteurs, l'adoption des nouvelles variétés et la formation des producteurs de niébé au Bénin a permis d'augmenter quantitativement et qualitativement la production. Les producteurs impliqués dans l'évaluation des performances à travers les Champs Ecoles Paysans, ont été formés sur la méthode de sélection et d'évaluation ainsi que des visites inter et intra villages ont été organisées pour permettre aux partenaires de comparer leurs parcelles à celles de leurs pairs. Cette hausse de la production a induit une amélioration des revenus des producteurs. Un résultat similaire chez les producteurs de niébé au Burkina Faso a été mis en évidence par (Rabe, Baoua, Sitou, \& Amadou, 2017). Ces auteurs montrent que la formation sur la maîtrise de l'itinéraire technique combinant variétés améliorées, le respect des écartements entre les plants, la protection des cultures et l'apports des fertilisants minéraux, a permis d'augmenter le rendement de niébé de $113 \%$ au Burkina Faso. De façon similaire Godtland et al (2004) ont constaté que l'amélioration des connaissances sur les pratiques de gestion intégrée des ravageurs a un impact positif sur la productivité de la pomme de terre au Pérou. A l'aide de données d'enquête, les auteurs ont évalué l'impact d'un programme pilote d'agriculture de formation des agriculteurs de la pomme de terre sur leur niveau de connaissance et sur la productivité des parcelles en production. Les résultats révèlent que les agriculteurs qui participent au programme ont beaucoup plus de connaissances sur les pratiques de lutte intégrée que ceux du groupe témoin non participant. Les auteurs trouvent également des preuves évocatrices selon lesquelles une meilleure connaissance des pratiques de lutte antiparasitaire intégrée contribue à améliorer considérablement la productivité de la production de pommes de terre. 
Les résultats de ces programmes de formation qui améliorent le niveau de connaissance ainsi que la productivité des parcelles ont plusieurs canaux de transmission. L'un des canaux est le taux d'adoption des techniques et des technologies enseignées. Gideon, et al 2017 montrent que la formation de producteurs de maïs à travers la fréquentation des ateliers agricoles a un impact positif sur l'intensité d'adoption des variétés améliorées de maïs au Ghana. Ces résultats sont renforcés par les travaux de Lauren \& Peter, (2017) qui montrent que parmi les facteurs qui influencent l'adoption de nouvelles variétés de soja chez les petits producteurs, il y a la formation sur les pratiques agricoles. En effet, selon eux, les déterminants influençant significativement l'adoption de variétés de soja améliorées chez les petits agriculteurs de l'est de l'Ouganda incluaient la participation des agriculteurs à la formation sur les pratiques agronomiques du soja, le nombre de visites d'un vulgarisateur, le rendement de la variété de soja et la taille du ménage.

Un autre canal par lequel les gains de productivité dans le secteur agricole peuvent apparaître, c'est celui de l'innovation. En effet la formation agricole peut contribuer au développement agricole en renforçant la capacité d'innover, la capacité d'introduire de nouveaux produits et processus socialement ou économiquement pertinents pour les petits exploitants agriculteurs et autres acteurs du secteur agricole (Spielman, Ekboir, Davis, \& Ochieng, 2008).

Des auteurs souligent cependant que les effets positifs de la formation sur les performances des agriculteurs peuvent être différenciés en fonction de leurs caractéristiques observables. En effet, dans une étude réalisée sur les producteurs de riz en Chine avec l'approche Champ Ecole Paysans a permis d'améliorer les connaissances des agriculteurs en matières de lutte contre les parasites et de la gestion de l'environnement (Mingliang, et al 2015). Toutefois, ces auteurs n'ont pas pu mettre en évidence un effet significatif sur la gestion des nutriments et les connaissances sur la culture. Mais concernant les performances des producteurs, ils montrent que les effets de l'approche Champ Ecole Paysans dans la rizicculture en Chine a des effets différenciés sur les performances des producteurs. Les effets sont plus faibles chez les femmes et les personnes âgées. Cette étude montre que les effets de l'approche Champ Ecole Paysans sont limités sur certains aspects liés à la gestion de l'activité de production d'une part et d'autre part les impacts sont différenciés selon le sexe et l'âge des producteurs.

Il est bon de noter aussi que la formation dans le milieu agricole peut se faire selon plusieurs approches dont l'approche Champ Ecole Paysans (CEP), l'approche formation et visite de terrain. D'une approche à l'autre, les résultats ne sont pas les mêmes. Des études ont montré l'efficacité de l'approche CEP comparativement à l'approche formation et visites de terrain. Cette efficacité repose sur la grande implication et la responsabilisation des 
premiers acteurs du monde paysan : les producteurs eux-mêmes (Williams \& Bembridge, 1990 ; Khosa, Van Averbek, Böhringer, \& Albertse, 2002 ; Okoth, et al., 2006). Au cours de la décennie 2000 des auteurs ont montré que cette approche Champ Ecole a beaucoup d'avantages dont l'augmentation des rendements des producteurs (Nabirye et al., 2002; Hakiza et al., 2004 ; van den Berg et Jiggins, 2007; Davis, 2008 ; Yoroba et al., 2011; Davis et al., 2012), la sensibilisation des producteurs et la diffusion des technologies éprouvées (van den Berg et Jiggins, 2007), l'émergence et la structuration des réseaux d'organisations paysannes (van den Berg et Jiggins, 2007). Certaines études ont montré que la formation contribue non seulement à l'améliroation du niveau de connaisssance des producteurs mais aussi elle participe à l'amélioration de leur niveau de vie. En Afrique de l'Est, la formation des agriculteurs à travers les champs écoles paysans a eu un impact positif et significatif sur la productivité des cultures et le revenu agricole par habitant (Kristin, et al., 2010).

La formation agricole contribue à l'améliroation des performances des producteurs. Les effets positifs de cette formation contribue à l'augmentation de la productivité des agriculteurs à travers l'amélioration de leur niveau de connaissance sur les pratiques et les techniques culturales, leur capacité d'innover et d'introduire de nouveaux processus économiquement pertinents dans leurs activités de production. Dès lors la formation et l'encadrement dans le milieu agricole favorise des gains de productivité chez les agriculteurs et une meilleure performance dans le milieu agricole. Est-ce que 1'appui conseil à l'attention des producteurs d'anacarde de Côte d'Ivoire a permis une hausse du rendement?

\section{Description du projet}

En 2013, le gouvernement ivoirien a adopté une réforme des filières coton et anacarde qui constituent une couche très importante de l'économie ivoirienne. La réforme prévoyait la mise en œuvre d'un encadrement dédié à l'ensemble des producteurs qui exercent dans la filière. L'objectif de celle-ci est de garantir la durabilité de la culture et d'améliorer la productivité et les revenus des producteurs. A cet effet, sur la base d'une convention cadre signée avec le Conseil Coton-Anacarde et le FIRCA, l'ANADER est chargée de s'assurer de : (i) La vulgarisation des variétés améliorées ; (ii) la formation aux bonnes pratiques agricoles (création et entretien des parcelles, opérations de récolte et post-récolte); (iii) l'identification des producteurs et des parcelles, l'élaboration des prévisions de récolte, et le suivi des statistiques de la production ; (iv) des pépiniéristes agréés seront chargés de la multiplication et de la commercialisation.

L'objectif de ce projet est d'assurer la formation des producteurs aux bonnes pratiques agricoles en vue d'accroître durablement la production, 
améliorer la qualité de la noix de cajou et leurs revenus. De manière spécifique, ce projet visait à : (i) assurer l'information, la sensibilisation et la formation des producteurs aux bonnes pratiques agricoles ; (ii) collecter des informations sur la production (superficie, rendement, âge du verger...) ; (iii) assurer un appui à la commercialisation primaire notamment par la sensibilisation ; (iv) rendre compte des résultats d'exécution de l'encadrement dédié par la production de rapports périodiques.

Dans l'optique d'atteindre les objectifs précités, les conseils diffusés par l'ANADER sur les bonnes pratiques agricoles portent essentiellement sur l'itinéraire technique de la culture de l'anacardier, les activités de récolte et post-récolte, et l'organisation des producteurs pour la commercialisation. Dans le but d'atteindre un maximum de producteurs, les outils de vulgarisation utilisés ont été variés. Il s'agit des champs écoles paysans(CEP), des parcelles de démonstration(PD), des unités de démonstration(UD) et de formation village $(\mathrm{FV})$.

En 2014, un accord a été signé entre les principales entités concernées par la mise en œuvre de ce projet à savoir l'autorité de régulation de la filière, le Fonds Interprofessionnel pour la Recherche et le Conseil Agricoles (FIRCA) ainsi que l'Agence National pour le Développement Rural (ANADER). Cette signature a marqué le début du projet dans lequel l'ANADER était chargé de vulgariser les variétés améliorées, de former aux bonnes pratiques agricoles (création et entretien des parcelles, opérations de récolte et poste récolte), d'identifier les producteurs et des parcelles, de suivre des statistiques de production, etc. Tout ce dispositif devrait permettre, in fine, d'améliorer les rendements, la qualité des noix et des paumes de cajou, de maîtriser des charges des producteurs et ainsi donc leurs revenus. La chaîne de changement attendu est telle que : la formation dans les bonnes pratiques va améliorer le niveau de connaissance des producteurs d'anacarde. Une fois le niveau de connaissance amélioré, l'on s'attend à ce que les producteurs suivent le protocole depuis la préparation de la parcelle jusqu'à la production en passant par l'entretien. Le Tableau 1 présente le contenu thématique de la formation. 
Tableau 1 : Thèmes et libellés

\begin{tabular}{|c|l|}
\hline Thèmes & Libellés \\
\hline 1 & Nettoyage du terrain \\
\hline 2 & Piquetage du terrain \\
\hline 3 & Trouaison et rebouchage \\
\hline 4 & Planting \\
\hline 5 & Acquisition de semence \\
\hline 6 & Semis direct \\
\hline 7 & Taille de formation \\
\hline 8 & Nettoyage de la parcelle \\
\hline 9 & Création de bande pare-feu \\
\hline 10 & Eclaircie d'une plantation d'anacardiers \\
\hline 11 & Elagage d'une plantation d'anacardiers \\
\hline 12 & Fertilisation d'une plantation d'anacarde \\
\hline 13 & Ramassage et séparation des fruits \\
\hline 14 & Séchage et triage des noix \\
\hline 15 & Condition de stockage \\
\hline 16 & Grainage \\
\hline & Source : Protocole de l'étude \\
\hline
\end{tabular}

Ce sont au total 16 thèmes qui ont été couverts par cet appui conseil. La formation couvre tout l'itinéraire : de la mise en place de la plantation jusqu'à la récolte.

\section{Données de l'étude}

Les données utilisées proviennent de l'enquête d'évaluation menée par l'ANADER du 04 au 14 juillet 2016. Cette collecte de données a concerné les cinq régions productrices de l'anacarde en Côte d'Ivoire : centre, centre-ouest, centre-est, ouest et nord. Dans les zones d'intervention de l'ANADER plusieurs localités bénéficiaires/non bénéficiaires ont été identifiées pour la constitution de l'échantillon des producteurs. Un sondage stratifié à deux degrés a permis de constituer l'échantillon des producteurs pour la conduite de l'enquête.

Les strates sont constituées par toutes les cinq (05) régions et les trentequatre (34) zones d'intervention de l'ANADER. Au premier degré, les localités qui constituent les unités primaires ont été tirées dans les zones proportionnellement au nombre de localités dans les Directions Régionales (05 régions de l'ANADER). Quant à la taille des localités à tirer, elle a été déterminée sur la base de la proportion des localités productrices d'Anacarde (27\%) au sein desquelles des producteurs ont été formés.

Partant de cette proportion, ils ont cherché un intervalle de confiance à $95 \%$ avec une marge de tolérance de plus ou moins $8 \%$. De cet intervalle, ils ont extrait la taille nécessaire pour obtenir cette précision. Cela a conduit à 
retenir 118 localités bénéficiaires tirées de façon aléatoire sur les 1530 localités bénéficiaires. A cet effectif, ont été ajoutées les localités non bénéficiaires d'où sera tiré le contrefactuel. En raison d'une localité par zone, ils ont obtenu 34 localités. Le choix de localités non bénéficiaires pour le contrefactuel s'est fait dans un souci de précaution du fait qu'il pourrait y avoir l'existence d'un "effet de contagion" par les bénéficiaires s'expliquant par leur proximité avec les non bénéficiaires. En tout, 152 localités ont été enquêtées. Le tirage des producteurs procède de la même logique que celle des localités. La proportion des producteurs d'Anacarde dans l'ensemble de la zone est estimé à 24\% (selon les statistiques du programme ANADERSTAT 2014). Ils ont recherché, comme précédemment, un intervalle de confiance de cette proportion à $95 \%$ avec une marge d'erreur de $3 \%$. Cette précision permet d'obtenir une taille de l'échantillon grâce à la formule suivante :

$$
\mathrm{n}=\frac{t^{2} \times p(1-p)}{\varepsilon^{2}} * d e f f .
$$

$\mathrm{n}$ est la taille de l'échantillon, $\boldsymbol{\varepsilon}$ la marge d'erreur, $\boldsymbol{p}$ la proportion de producteurs d'Anacarde formés, $t$ le quantile d'ordre $\alpha=5 \%$ de la loi normale et $\boldsymbol{d e f f}$, l'effet de sondage. Pour $\mathrm{p}=24 \%$ et $\operatorname{deff}=2$, ils ont obtenu la taille de 1644 producteurs pour l'ensemble des 34 zones du projet. En ajoutant à ce nombre l'effectif de 10 producteurs non bénéficiaires dans chacune des localités non bénéficiaires (soit 340 producteurs), la taille finale des producteurs à enquêter s'est élevée à 1984 producteurs. Le Tableau 2 présente la répartition de l'échantillon final de l'étude.

Tableau 2 : Répartition des producteurs bénéficiaires et des non bénéficiaires par région

\begin{tabular}{|l|c|c|c|c|c|}
\hline $\begin{array}{l}\text { Direction } \\
\text { Régionale }\end{array}$ & $\begin{array}{c}\text { Nombre de } \\
\text { zones }\end{array}$ & $\begin{array}{c}\text { Taille } \\
\text { d'échantillon } \\
\text { localité }\end{array}$ & $\begin{array}{c}\text { Nombre } \\
\text { producteurs } \\
\text { bénéficiaires }\end{array}$ & $\begin{array}{c}\text { Nombre } \\
\text { producteurs } \\
\text { non } \\
\text { bénéficiaires }\end{array}$ & $\begin{array}{c}\text { Taille } \\
\text { d'échantillon } \\
\text { producteur }\end{array}$ \\
\hline Centre & 10 & 42 & 543 & 100 & 643 \\
\hline Centre ouest & 7 & 20 & 140 & 70 & 210 \\
\hline Centre-est & 7 & 33 & 319 & 70 & 389 \\
\hline Nord & 8 & 41 & 478 & 80 & 558 \\
\hline Ouest & 2 & 16 & 164 & 20 & 184 \\
\hline Total général & $\mathbf{3 4}$ & $\mathbf{1 5 2}$ & $\mathbf{1 6 4 4}$ & $\mathbf{3 4 0}$ & $\mathbf{1 9 8 4}$ \\
\hline
\end{tabular}

Source : Agence nationale d'Appui au développement rural

\section{Choix de la méthodologie de l'évaluation de l'impact de l'appui conseil sur les rendements des parcelles}

L'évaluation de l'effet de l'appui conseil sur les performances des producteurs s'est faite en appliquant les méthodes micro économétriques. La méthode choisie dans cette analyse est la méthode d'appariement sur scores de propension. Selon la littérature, l'on peut estimer l'effet causal du projet par diverses méthodes. La méthode retenue est la méthode du noyau (Heckman, Ichimura, \& Todd, 1998). C'est une méthode non paramétrique 
d'estimation de l'effet causal. Elle utilise une moyenne pondérée de tous les non-bénéficiaires pour construire la correspondance contrefactuelle pour chaque bénéficiaire du projet ${ }^{3}$. Les poids attribués dépendent de la distance entre chaque producteur du groupe des non bénéficiaires et l'observation du producteur du groupe des bénéficiaires pour laquelle le contrefactuel est estimé. Un estimateur consistant du résultat contrefactuel est noté :

$\hat{E}\left[Y \mid \hat{\pi}\left(X_{i}\right), T=0\right]=\sum_{j \in C} w_{i j} Y_{j}^{C}$

Avec $w_{i j}=\frac{K\left[\frac{\hat{\pi}\left(X_{j}\right)-\hat{\pi}\left(X_{i}\right)}{h_{n}}\right]}{\sum_{k \in C} K\left[\frac{\hat{\pi}\left(X_{k}\right)-\hat{\pi}\left(X_{i}\right)}{h_{n}}\right]}$ la pondération, où $K($.$) est une fonction noyau,$ $h_{n}$ est la fenêtre du noyau $K$. L'effet moyen de la formation sur le rendement des producteur d'anacarde de Côte d'Ivoire est donné par :

$\Delta_{K M}^{A T T}=\frac{1}{N^{T}} \sum_{i \in T}\left\{Y_{i}^{T}-\frac{\sum_{j \in C} Y_{j}^{C} K\left[\frac{\hat{\pi}\left(X_{j}\right)-\widehat{\pi}\left(X_{i}\right)}{h_{n}}\right]}{\sum_{k \in C} K\left[\frac{\hat{\pi}\left(X_{k}\right)-\widehat{\pi}\left(X_{i}\right)}{h_{n}}\right]}\right.$

Avec les indices $T, C$ désignant les producteurs du groupe des bénéficiaires et les bénéficiaires du groupe des non bénéficiaires. $N$ désigne l'effectif total. Heckman, Ichimura, \& Todd, (1998) montrent que, sous certaines hypothèses de régularité, cet estimateur est convergent, asymptotiquement normal, avec une vitesse de convergence en racine carrée de $\mathrm{N}$.

\section{Résultats de l'évaluation de l'impact du projet Estimations des scores de propension}

Les facteurs observables utilisés dans l'estimation des scores de propension sont présentés dans le Tableau 3. Ces variables ont été choisies car elles peuvent influencer la performance des producteurs sans avoir une relation a priori avec la participation au programme de formation des producteurs. De plus l'option retenue pour tester la qualité de l'appariement devrait conforter cette sélection de variables. Ce qui est le cas (cf. Tableau 4).

\footnotetext{
${ }^{3}$ Sachant les facteurs observables des bénéficiaires du projet
} 
Tableau 3 : Variables retenues dans l'estimation des scores de propension

\begin{tabular}{|l|l|}
\hline Variables & Type \\
\hline Nombre d'années de la pratique de la culture d'anacarde & Quantitative \\
\hline Appartenance à une coopérative & Qualitative \\
\hline Niveau d'instruction des producteurs & Qualitative \\
\hline Superficie totale cultivée & Quantitative \\
\hline Utilisation d'intrants agricoles & Qualitative \\
\hline Utilisation de matériel végétal & Qualitative \\
\hline Accès au financement & Qualitative \\
\hline Mode de possession de la parcelle & Qualitative \\
\hline Age du producteur & Quantitative \\
\hline Sexe du producteur & Qualitative \\
\hline
\end{tabular}

Source : Les auteurs

Les scores de propensions sont donnés par les valeurs prédites des probabilités de participation au programme sachant les observables retenues. Ce score compris entre 0 et 1 est calculé pour chaque producteur de la base. La Figure représente la distribution des scores de propension dans les deux groupes de producteurs : les bénéficiaires et les non bénéficiaires.

Figure 1: Distribution des scores de propension avant l'appariement

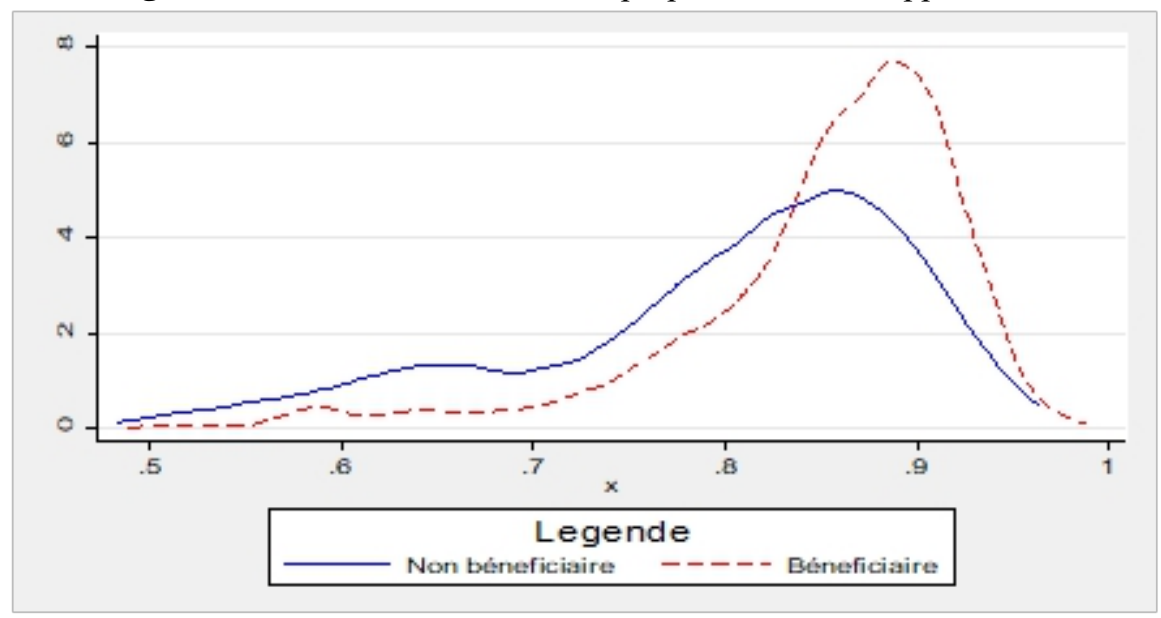

Source : Les auteurs

Cette Figure 1 montre que les deux courbes se chevauchent. Nous pouvons donc affirmer qu'il existe un support commun sur lequel l'on peut apparier les bénéficiaires et les non bénéficiaires.

\section{Détermination du support commun}

Le support commun permet de trouver des producteurs non bénéficiaires qui ont pratiquement les mêmes scores de propension que les producteurs bénéficiaires. Le support commun représente donc la zone où la courbe des scores de propensions des bénéficiaires et celle des non 
bénéficiaires se chevauchent parfaitement, Le support commun obtenu dans notre cas est $[0,488 ; 0,989]$. La distribution des scores sur le support commun, selon que le producteur ait bénéficié ou non du programme est donné par la Figure 2. On remarque que la zone de support est très étendue ; le support prend en compte un très grand nombre de producteurs $(95 \%)$ de notre base qui sont comparables.

Figure 2: Distribution des scores de propension sur le support commun

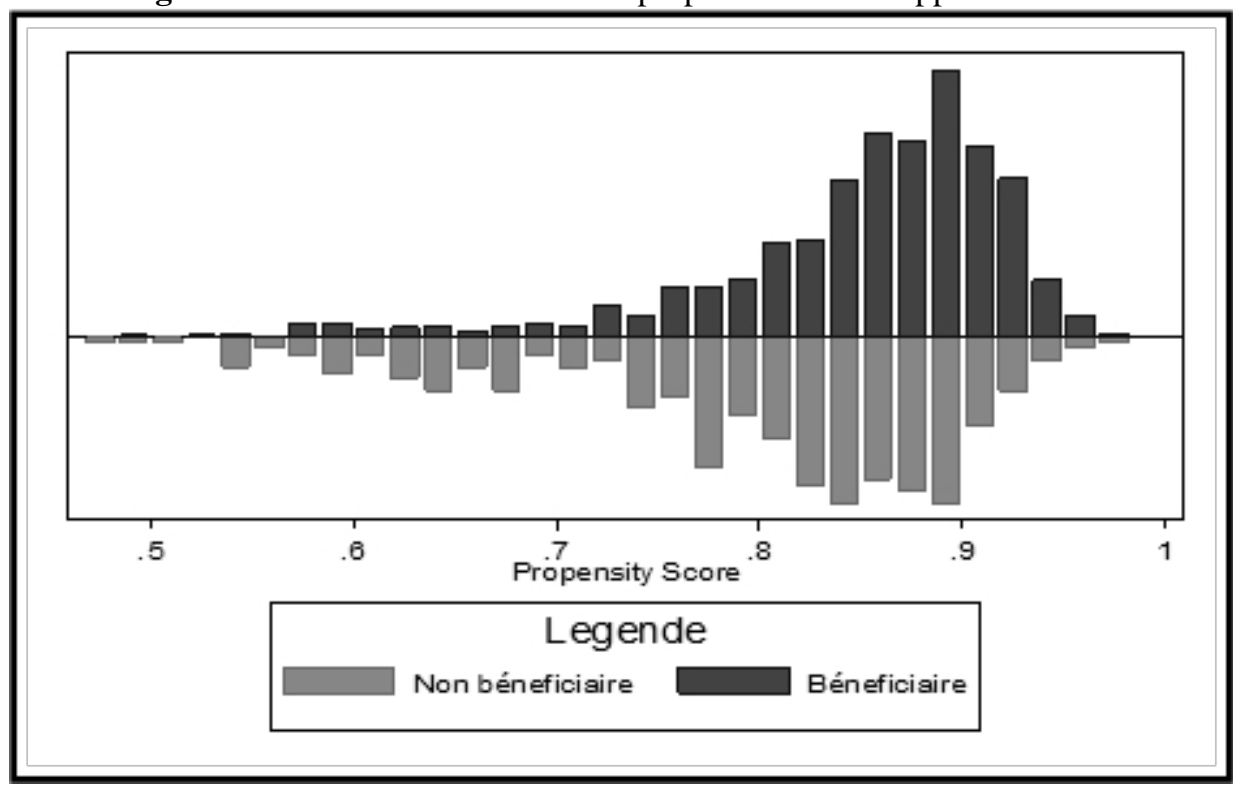

Source : Les auteurs

Par ailleurs, afin de s'assurer que les caractéristiques observables retenues expliquent réellement la sélection des bénéficiaires, nous testons la qualité de l'appariement.

\section{Test de qualité de l'appariement}

La qualité de l'appariement permet d'apprécier la fiabilité de l'estimation du score de propension. Elle est appréhendée à travers la capacité du score de propension à résumer les caractéristiques observables utilisées pour l'appariement. Concrètement, pour vérifier cette hypothèse, nous devrions présenter à nouveau les caractéristiques observables et montrer qu'elles sont indépendantes de l'accès au groupe de traitement pour les individus du support commun. Mais pour contourner cela, la littérature nous propose plusieurs "balancing tests" ou tests de la propriété du score équilibrant qui permettent de vérifier si les caractéristiques individuelles moyennes ne diffèrent pas entre le groupe de contrôle et le groupe de traitement. Le test utilisé ici fait une régression de chaque variable explicative sur la variable de participation au projet et donne le t-test. S'il n'est pas 
significatif, il n'y a pas de différence significative entre le groupe de traitement et le groupe de contrôle pour la variable considérée. La régression est faite avant l'appariement sur tout l'échantillon, et après appariement sur le support commun en moindres carrés pondérés. Les résultats du diagnostic sont présentés dans le Tableau 4.

Tableau 4: Résultats du test de la qualité de l'appariement

\begin{tabular}{|c|c|c|c|c|c|}
\hline \multirow{2}{*}{$\begin{array}{l}\text { Variables ayant } \\
\text { servi pour } \\
\text { l'appariement }\end{array}$} & \multirow[b]{2}{*}{ Echantillons } & \multicolumn{2}{|c|}{ Statistiques du test } & \multirow[t]{2}{*}{ P-value } & \multirow{2}{*}{$\begin{array}{c}\text { Différence après } \\
\text { appariement }\end{array}$} \\
\hline & & Traités & Contrôle & & \\
\hline \multirow{2}{*}{ Sexe } & Non-apparié & 1,13 & 1,13 & 0,05 & \multirow[t]{2}{*}{ Non significative } \\
\hline & Apparié & 1,13 & 1,14 & 0,33 & \\
\hline \multirow{2}{*}{ Age } & Non-apparié & 48,88 & 48,16 & 0,00 & \multirow[t]{2}{*}{ Non Significative } \\
\hline & Apparié & 48,81 & 48,66 & 0,83 & \\
\hline \multirow{2}{*}{$\begin{array}{l}\text { Nombre d'années } \\
\text { de la pratique de la } \\
\text { culture d'anacarde }\end{array}$} & Non-apparié & 13,59 & 11,42 & 0,09 & \multirow[t]{2}{*}{ Non -significative } \\
\hline & Apparié & 13,51 & 13,31 & 0,05 & \\
\hline \multirow{2}{*}{$\begin{array}{l}\text { Appartenance à } \\
\text { une coopérative }\end{array}$} & Non-apparié & 0,15 & 0,11 & 0,00 & \multirow[t]{2}{*}{ Non significative } \\
\hline & Apparié & 0,15 & 0,12 & 0,06 & \\
\hline \multirow{2}{*}{ Education } & Non-apparié & 0,26 & 0,33 & 0,87 & \multirow[t]{2}{*}{ Non significative } \\
\hline & Apparié & 0,26 & 0,28 & 0,31 & \\
\hline \multirow{2}{*}{$\begin{array}{l}\text { Superficie totale } \\
\text { cultivée }\end{array}$} & Non-apparié & 3,95 & 3,40 & 0,53 & \multirow[t]{2}{*}{ Non Significative } \\
\hline & Apparié & 3,94 & 3,62 & 0,11 & \\
\hline \multirow{2}{*}{$\begin{array}{l}\text { Utilisation } \\
\text { d'intrants agricoles }\end{array}$} & Non-apparié & 0,37 & 0,29 & 0,00 & \multirow[t]{2}{*}{ Non significative } \\
\hline & Apparié & 0,37 & 0,34 & 0,15 & \\
\hline \multirow{2}{*}{$\begin{array}{l}\text { Utilisation de } \\
\text { matériel végétal }\end{array}$} & Non-apparié & 0,01 & 0,01 & 0,00 & \multirow[t]{2}{*}{ Non significative } \\
\hline & Apparié & 0,01 & 0,01 & 0,34 & \\
\hline \multirow{2}{*}{$\begin{array}{l}\text { Accès au } \\
\text { financement }\end{array}$} & Non-apparié & 0,03 & 0,01 & 0,00 & \multirow[t]{2}{*}{ Non significative } \\
\hline & Apparié & 0,03 & 0,01 & 0,81 & \\
\hline \multirow{2}{*}{$\begin{array}{l}\text { Mode de } \\
\text { possession- dons }\end{array}$} & Non-apparié & 0,17 & 0,19 & 0,00 & \multirow[t]{2}{*}{ Non significative } \\
\hline & Apparié & 0,17 & 0,18 & 0,32 & \\
\hline \multirow{2}{*}{$\begin{array}{l}\text { Mode de } \\
\text { possession-legs }\end{array}$} & Non-apparié & 0,14 & 0,19 & 0,00 & \multirow[t]{2}{*}{ Non significative } \\
\hline & Apparié & 0,14 & 0,14 & 0,62 & \\
\hline \multirow{2}{*}{$\begin{array}{l}\text { Mode de } \\
\text { possession-héritage }\end{array}$} & Non-apparié & 0,63 & 0,44 & 0,00 & \multirow[t]{2}{*}{ Non significative } \\
\hline & Apparié & 0,63 & 0,61 & 0,27 & \\
\hline
\end{tabular}

Source : Nos calculs

La statistique du test indique évalue la différence statistique entre les échantillons avant et après appariement. Il ressort de ce tableau que la différence après appariement n'est pas significative en considérant la quasitotalité des variables retenues. Ainsi, les résultats montrent que l'on pourrait retenir qu'après appariement, les scores de propension moyens ne sont pas statistiquement différents au seuil de 5\%. Ce qui veut dire que ces variables ne diffèrent pas en moyenne entre le groupe de contrôle et le groupe de traitement. L'appariement est donc d'une qualité suffisante pour chacune des variables. 


\section{Evaluation de l'effet du programme sur le rendement des producteurs}

Le Tableau 5 présente les résultats de l'évaluation de l'impact du projet sur le rendement des producteurs d'anacarde par la méthode du noyau.

Tableau 5: Résultats des estimations sur le rendement des producteurs d'anacarde

\begin{tabular}{|l|c|c|}
\hline Méthodes & $\begin{array}{c}\text { Effet moyen }{ }^{1} \text { en } \\
\mathrm{kg} / \mathrm{h}\end{array}$ & $\begin{array}{c}\% \text { augmentation } \\
\text { par rapport aux non } \\
\text { bénéficiaires }\end{array}$ \\
\hline \multicolumn{1}{|c|}{ Variable de résultat : Le rendement : la quantité produite à l'hectare $\mathrm{kg} / \mathrm{h}$} \\
\hline Méthode de noyau & $\begin{array}{c}141,96 \\
(4,51)\end{array}$ & 22,4 \\
\hline $\begin{array}{l}{ }^{1} \text { Différence moyenne entre le rendement de producteurs bénéficiaires et des non } \\
\text { bénéficiaires ; () t statistique } \\
\text { Source : Nos calculs }\end{array}$
\end{tabular}

Le projet a eu un impact positif sur le rendement des producteurs d'anacarde. La hausse du rendement due à l'appui-conseil est de $141 \mathrm{~kg} / \mathrm{h}$ en moyenne. La formation a permis d'augmenter la production d'anacarde à l'hectare de $22,4 \%$ en moyenne. Ce projet de formation a effectivement contribué à améliorer significativement les performances des producteurs d'anacarde de Côte d'Ivoire. Ce résultat montre que la formation et l'encadrement des producteurs a un impact positif sur leur performance. Un résultat similaire a été mis en évidence par Kristin et al., (2010) en Afrique de l'Est. En effet les auteurs ont montré que la formation des agriculteurs à travers les champs écoles paysans a eu un impact positif sur la productivité des cultures et le revenu agricole par habitant. Tout comme Maïga et al, (2017) qui a montré que les sous-performances des producteurs de riz au Niger peuvent s'expliquer par l'inefficience de l'encadrement technique. Ainsi l'encadrement, l'appui conseil apporté aux producteurs d'anacarde en Côte d'Ivoire a entraîné une hausse du rendement. Théoriquement, l'amélioration du niveau de connaissance des producteurs a induit une bonne connaissance des bonnes pratiques. L'amélioration du niveau de connaissance a eu un impact sur l'efficacité des producteurs. Cette efficacité s'est traduite par une meilleure productivité qui a entrainé une amélioration des rendements. Toutefois, de façon rigoureuse, les gains de produtivité ne sauraient être exlcusivement le résultat de ce projet dans la mesure où plusieurs actions antérieures ou des politiques similaires pourraient aussi avoir contribué à la consolidation de ces acquis de la formation reçue par les producteurs d'anacarde dans le cadre de ce projet. Aussi, cette évaluation n'a pas pu isoler la compossante spécifique du programme d'appui selon les thèmes abordés qui a eu le plus d'impact. L'évaluation a été faite globalement sur le packquage offert aux producteurs dans le cadre de ce projet. 


\section{Contrôle de la robustesse des résultats de l'estimation de l'effet causal}

Deux approches ont été utilisées pour réaliser le contrôle de la robustesse de ces résultats. La première a consisté en la mise en œuvre d'autres méthodes d'estimations par appariement. La seconde approche a été conduite en appliquant directement un appariement en dehors des scores de propension, L'approche consiste à appliquer un appariement direct du voisinage le plus proche au lieu d'estimer d'abord l'équation du score de propension. Les estimations donnent d'apprécier la robustesse des résultats de l'impact du projet de conseil agricole sur les rendements de l'anacarde en Côte d'Ivoire.

$\mathrm{S}$ 'agissant de la première approche les résultats sont présentés dans le Tableau 6, Toutes les estimations confirment l'impact positif du projet sur le rendement de l'anacarde avec un effet moyen se situant dans le même ordre de grandeur que la méthode retenue.

Tableau 6: Résultats des estimations de l'effet moyen avec d'autres méthodes d'appariement

\begin{tabular}{|l|c|c|}
\hline Méthodes & $\begin{array}{c}\text { Effet moyen }{ }^{1} \text { en } \\
\mathrm{kg} / \mathrm{h}\end{array}$ & $\begin{array}{c}\text { \% augmentation } \\
\text { par rapport aux non } \\
\text { bénéficiaires }\end{array}$ \\
\hline \multicolumn{2}{|c|}{ Variable de résultat : Le rendement : la quantité produite à l'hectare $\mathrm{kg} / \mathrm{h}$} \\
\hline Appariement exact avec groupement & $\begin{array}{c}166,23 \\
(4,15)\end{array}$ & 26,8 \\
\hline Appariement avec stratification & $\begin{array}{l}166,7 \\
(6,52)\end{array}$ & 26,8 \\
\hline Plus proche voisin & $\begin{array}{c}175,05 \\
(4,48)\end{array}$ & 26,9 \\
\hline Appariement avec un rayon & $\begin{array}{c}172,35 \\
(4,63)\end{array}$ & 26,8 \\
\hline
\end{tabular}

${ }^{1}$ Différence moyenne entre le rendement de producteurs bénéficiaires et des non bénéficiaires; () t statistique

Source : Nos calculs

L'estimation directe de l'effet donne des résultats en ligne avec les résultats obtenus avec la méthode des noyaux (cf. Tableau 7).

Tableau 7: Résultats de l'estimation directe de l'effet causal

\begin{tabular}{|l|l|c|c|c|}
\hline $\begin{array}{l}\text { Variable dépendante : Rendement } \\
(\mathrm{kg} / \mathrm{h})\end{array}$ & Coefficient & $\mathrm{z}$ & $\mathrm{p}>|\mathrm{z}|$ & $\begin{array}{c}\text { Intervalle de confiance } \\
95 \%\end{array}$ \\
\hline Effet moyen du projet & 181,17 & 4,65 & 0,00 & {$[104,79 ; 257,56]$} \\
\hline
\end{tabular}

Un impact positif de $181,18 \mathrm{Kg} /$ ha de la participation au programme de formation dédié aux producteurs d'anacarde est observé à un niveau de significativité de $5 \%$. Ce résultat vient confirmer les résultats obtenus précédemment. 


\section{Conclusion et recommandations}

Cette analyse visait à évaluer l'impact du projet de conseil agricole sur les performances des producteurs d'anacarde de Côte d'Ivoire. L'étude a évalué l'impact de ce projet sur le rendement des producteurs à partir de la méthode d'appariement sur score de propension. Les données utilisées proviennent de l'enquête réalisée par le département suivi et évaluation de l'Agence National pour le Développement Rural, qui s'est vu confié la mise en œuvre de cet appui conseil au profit des producteurs d'anacarde en Côte d'Ivoire. La taille de l'échantillon est de 1984 producteurs dont 340 non bénéficiaires répartis sur l'ensemble du territoire.

Il ressort que les rendements de ces producteurs formés ont augmenté de l'ordre de 22,4\% en moyenne avec ce projet. En sommes, le conseil agricole a permis d'améliorer les performances des producteurs d'anacarde. De ce qui précède, qu'il nous soit permis de formuler les recommandations suivantes pour l'amélioration des effets du conseil agricole dédié aux producteurs d'anacarde à l'endroit de l'ANADER l'agence principale d'encadrement et des centres de recherche, des partenaires techniques et financiers, du ministère en charge de l'agriculture et des producteurs bénéficiaires.

Une extension du programme de formation des producteurs pourrait permettre une plus grande performance nationale dans la production d'anacarde: il s'agira pour les partenaires techniques et financiers d'augmenter leur appui financier et à l'Etat de créer des espaces budgétaires pour le financement de tel projet afin de toucher le plus grand nombre des producteurs.

L'effet positif du projet sur l'augmentation des rendements des parcelles nous permet de recommander à l'ensemble des acteurs, aux bailleurs et l'ANADER) d'étendre le programme de formation à l'ensemble des producteurs d'anacarde afin d'accroitre le rendement national.

A l'endroit du ministère en charge de l'agriculture, il sera important de soutenir la vulgarisation des champs écoles paysans (CEP), des parcelles de démonstration (PD), des unités de démonstration (UD) et de formation village (FV) et de mettre à disposition des moyens matériels et humains nécessaires. Il a été montré dans la revue de littérature que l'approche Champ école paysans est plus efficace en matière de résultats de la formation et de l'encadrement des paysans sur les rendements agricoles (Williams \& Bembridge, 1990 ; Khosa, Van Averbek, Böhringer, \& Albertse, 2002 ; Okoth, et al., 2006).

A l'endroit des centres de recherche, il s'agira de poursuivre la recherche dans les bonnes pratiques culturales en fonction des types de sols et d'appuyer leur vulgarisation et leur promotion dans le milieu paysan.

Aux producteurs d'anacarde, il leur sera utile de poursuivre l'application des formations reçues de façon rigoureuse pour de meilleurs rendements. De plus, les producteurs devraient faire remonter les informations 
sur les pratiques pour une meilleure adaptation de la formation dans les zones agro climatiques.

\section{References:}

1. Aly, D., Ahouansou, R. H., Mama, V. J., Olou, D., \& Agli, C. (2017). Evaluation et selection participative des varietes ameliorees de niebe en milieu rural dans le departement de couffo au Benin. African Crop Science Journal, 25(4), 509-520.

2. Binam, J. N., Gockowski, J., \& Nkamleu, G. B. (2008). Technical efficiency and productivity potential of cocoa farmers in West African countries. The Developing Economies, 46(3), 242-263.

3. Brodaty, T., Crépon, B., \& Fougère, D. (2007). Les méthodes microéconométriques d'évaluation et leurs applications aux politiques actives de l'emploi. Economie \& prévision, 177(1), 93-118. Récupéré sur https://www.cairn.info/revue-economie-et-prevision-2007-1page-93

4. Conseil Coton Anacarde. (2018, Octobre 14). www.cashewconvention.com/wcc2018/presentation/french/Adam_Re cent_evolution_of_the_Cashew_industries_in_Cote_d_Ivoire.pdf. Récupéré sur www.cashewconvention.com/wcc2018: http://www.cashewconvention.com/wcc2018/presentation/french/Ada $\underline{m \_ \text {Recent_evolution_of the_Cashew_industries_in_Cote_d_Ivoire.p }}$ df

5. Davis, K. E. (2008). Extension in Sub-Saharan Africa: Overview and assessment of past and current models, and future prospects. Journal of International Agricultural and Extension Education, 15(3), 15 - 28.

6. Davis, K., Nkonya, E., Kato, E., Mekonnen, D., Odendo, M., Miiro, R., \& J., N. (2012). Impact of Farmer Field Schools on Agricultural Productivity and Poverty in East Africa. World Development(40), 402 $-413$.

7. Erin, M, G,, Elisabeth, S, Alain, d, J,, Rinku, M, \& Oscar, O, (2004), The Impact of Farmer Field Schools on Knowledge and Productivity: A Study of Potato Farmers in the Peruvian Andes, Economic Development and Cultural Change, 53(1), 63-92,

8. Fougère, D. (2010). Les méthodes économétriques d'évaluation. Revue française des affaires sociales, 105-128. Récupéré sur https://www.cairn.info/revue-francaise-des-affaires-sociales-2010-1page-105.htm.

9. Gideon, D.-A., Joshua, A. B., Dennis, S. E., \& Franklin, N. M. (2017). Adoption of Improved Maize Variety among Farm Households in the Northern Region of Ghana. Cogent Economic \& Finance. doi: http://doi.org/10.1080/23322039.2017.1416896 
10. Godtland, E. M., Sadoulet, E., Janvry, A. D., Murgai, R., \& Ortiz, O. (2004). The impact of farmer field schools on knowledge and productivity: A study of potato farmers in the Peruvian Andes. Economic development and cultural change, 53(1), 63-92.

11. Hakiza, J. J., Odogola, W., Mugisha, J., Semana, A., Nalukwago, J., Okoth, J., \& E., A. (2004). Challenges and prospects of disseminating technologies through farmer field schools: Lessons learnt based on experience from Uganda. Uganda Journal of Agricultural Sciences, 9, 163 - 175.

12. Heckman, J. J., Ichimura, H., \& Todd, P. (1998). Matching as an econometric evaluation estimator. The review of economic studies, 65(2), 261-294.

13. Khosa, T, Van Averbek, W, Böhringer, R,, \& Albertse, E, (2002), Enriching the Traning \& visit approach to technology transfer by fusing the Roles of Researchers and Village Extension Worker, 18th annual conference of the Association for International Agriculture and Extension Education, 26-30 May (pp, 188-195), Durban, South Africa : College Station A\&M University,

14. Kristin, D, Ephraim, N, Edward, K, Daniel, A, M,, Martins, O, Richard, M,, \& Jackson, N, (2010), Impact of Farmer Field Schools on Agricultural Productivity and Poverty in East Africa, IFPRI Discussion Paper 00992,

15. Lauren, M., \& Peter, W. P. (2017, mai Lundi 08 janvier 2018). Determinants of adoption of improved soybean varieties among smallholder farmers in eastern uganda.

16. MAG-SDR, (2010), Etude sur la mise en place d'un dispositif intégré d'appui conseil pour le développement rural au Niger, Niamey: Sécrétariat exécutif comité interministériel de pilotage de la stratégie de développement rural,

17. Maïga, I. M., Himadou, A. A., Haougui, A., Souleymane, A., \& Ibro, G. (2017). Normes techniques et pratiques locales des producteurs dans les perimetres rizicoles irrigues de toula et de bonfeba au Niger. African Crop Science Journal, 25(4), 441-456.

18. Mingliang, G., Xiangping, J., Jikun, H., Krishna, B. K., \& Nicholas, E. B. (2015). Farmer field school and farmer knowledge acquisition in rice production: Experimental evaluation in China. Agriculture, Ecosystems \& Environment, 209, 100-107. doi:https://doi.org/10.1016/j.agee.2015.02.011.

19. Ministère de l'Intégration Africaine et des Ivoiriens de l'Extérieur. (2018, Octobre 14). www.integration.ci/Bookshelf/dossiereconomique/FICHESGENERA LES.pdf. 
www.integration.ci/Bookshelf/dossiereconomique/:

http://www.integration.ci/Bookshelf

20. Nabirye, J., Nampala, P., Ogenga-Latigo M., W., Kyamanywa, S., Wilson, H., Odekec, (2003). Farmer-participatory evaluation of cowpea integrated pest management (IPM) technologies in Eastern Uganda. Crop Protection 22.

21. Okoth, J,, Braun, A,, Delve, R,, Khamaala, H,, Khisa, G, \& Thomas, J, (2006), The emergence of Farmer Field Schools Networks in Eastern Africa, Cali, Colombia, IFPRI, Washington, 10 P,: Research Workshop on Collective Action and Market Acces for Smallholders, 2-5 October 2006,

22. Rabe, M, Baoua, I,, Sitou, L, \& Amadou, L, (2017), Champ école paysan, une approche participative pour l'amélioration du rendement du Niébé, Agronomie Africaine, sp, 29(2), 1-9

23. Spielman, D. J., Ekboir, J., Davis, K., \& Ochieng, C. M. (2008). An innovation systems perspective on strengthening agricultural education and training in sub-Saharan Africa. Agricultural systems, 98(1), 1-9.

24. Van Den Berg, H., \& Jiggins, J. (2007). Investing in Farmers-The Impacts of Farmer Field Schools in Relation to Integrated Pest Management. World development 35:

25. Williams, J, \& Bembridge, T, (1990), Training and visit (T\&V) extension: A model for Southern Africa, South African Journal of Agriculture Extension, 19, 23-30,

26. Yorobe, J. M., Rejesus, R. M., \& Hammig, M. D. (2011). Insecticide use impacts of Integrated Pest Management (IPM) Farmer Field Schools : Evidence from onion farmers in the Philippines. Agricultural Systems, 104(7), 580 - 587.

27. Zepeda, L. (2001). Agricultural investment, production capacity and productivity. Dans FAO, Agricultural Investment and Productivity in Developing Countries (pp. 3-20). Rome: FAO economic and social development paper. 